\title{
Excessive supraventricular activity and risk of atrial fibrillation in patients with cryptogenic ischaemic stroke
}

\author{
Milan Maretta ${ }^{1}$, Miloš Šimurda $^{2}$, Norbert Leško ${ }^{1}$, Miroslav Gbúr ${ }^{2}$, Matej Škorvánek ${ }^{1}$, Silvia Mišíková \\ Zuzana Gdovinová ${ }^{1}$ \\ ${ }^{1}$ Department of Neurology, P.J. Safarik University, Kosice, Slovakia \\ ${ }^{2} 1^{\text {st }}$ Department of Cardiology, Eastern Slovak Cardiovascular Institute and P.J. Safarik University, Kosice, Slovakia
}

\begin{abstract}
Introduction. Atrial fibrillation (AF) is one of the leading causes of ischaemic stroke. However, screening for AF is often time-consuming in clinical practice. Therefore, the determination of an appropriate marker to detect the presence of AF would improve the diagnostic process.

Objective. The aim of the current study was to evaluate the efficacy of prolonged and early inpatient event Holter monitoring in the detection of AF in patients with ESUS-related cryptogenic ischaemic stroke (CIS), and to determine the possible relationship between excessive supraventricular activity and AF detection.

Material and methods. All consecutive patients with documented cerebral or cerebellar infarction were included. The diagnostic work-up included brain neuroimaging (CT/MRI), ultrasound of the carotid and vertebral arteries, admission ECG followed by 24 hours of Holter monitoring, and transthoracic echocardiography. The 24-hour Holter ECG was analysed, and supraventricular ectopic activity (supraventricular extrasystoles, runs and pairs of supraventricular extrasystoles) was recorded in all patients. If these examinations did not reveal the cause of ischaemic stroke, the patients underwent subsequent prlonged 14-day event Holter recorder monitoring. Results. We included 48 patients (mean age $69.9 \pm 8.5$ years, $60.4 \%$ men) who had been diagnosed with CIS. Of these 48 patients, atrial fibrillation was detected in seven (14.6\%) during the prolonged 14-day Holter event monitoring. Patients with newly diagnosed atrial fibrillation had a higher burden of supraventricular ectopic activity. The number of supraventricular extrasystoles (SVES) per hour, as well as the number of $S V$ pairs and SV runs, was significantly higher in patients with new onset $\operatorname{AF}(p<0.022 ; p<0.043 ; p<0.022)$.

Conclusions. In our study, we confirmed that prolonged ECG event Holter monitoring in patients with CIS-ESUS subtype led to a higher rate of AF detection. Likewise, frequent supraventricular ectopic activity predicted the development of AF.
\end{abstract}

Key words: cryptogenic ischaemic stroke, atrial fibrillation, supraventricular ectopic activity, Holter ECG monitoring (Neurol Neurochir Pol 2022; 56 (1): 75-80)

\section{Introduction}

At least one-quarter of all ischaemic strokes have an unknown or undetected cause, and these cases are termed 'cryptogenic' [1]. However, some ischaemic strokes labelled as cryptogenic have been found to be caused by atrial fibrillation (AF) which was not detected by conventional diagnostic work-up, and these patients in the end had a cardio-embolic aetiology $[2,3]$. AF in patients with a first-ever stroke has been found to be a predictor of 30-day and 1-year mortality; likewise, this arrythmia increases the risk of stroke recurrence within the first year [4].

Screening for occult AF is therefore necessary in these patients. The presence of atrial ectopic beats has been found

Address for correspondence: Milan Maretta, Department of Neurology, Pavol Jozef Safarik University and L. Pasteur University Hospital, Trieda SNP 1, 04011 Kosice, Slovakia; e-mail: milan.maretta@gmail.com 
to be associated with an increased risk of ischaemic stroke in patients without previous cardiovascular events (i.e. stroke, myocardial infarction) [5]. Additionally, frequent atrial ectopic activity has been found to be related to a higher likelihood of new-onset AF and a higher prevalence of dementia and mild cognitive impairment, independently of stroke $[6,7]$. Several studies have confirmed possible cardiac biomarkers as predictors of AF, namely left atrial diameter and frequent atrial premature contraction [8]. Novel biomarkers suggesting an increased risk of AF include echocardiographic parameters (i.e. a left atrial volume index $>32 \mathrm{~mL} / \mathrm{m} 2$, and an abnormal left atrial longitudinal strain) or serum biomarkers (i.e. B type natriuretic peptide and troponin concentration) [9].

The aim of our study was to determine the usefulness of prolonged electrocardiogram (ECG) Holter monitoring, and to correlate supraventricular ectopic activity with new onset of $\mathrm{AF}$ as a predictor of $\mathrm{AF}$ development.

\section{Material and methods}

In all patients admitted to the Department of Neurology at University Hospital L. Pasteur with suspected stroke, brain computed tomography (CT) or magnetic resonance imaging (MRI) was performed to confirm the diagnosis of stroke. Only patients with brain infarct confirmed by CT or MRI were included in the study, and patients with transitory ischaemic attack (TIA) were excluded. Subsequently, laboratory tests (biochemistry, coagulation panel), admission ECG and 24-hour Holter monitoring (DMS 300-4L, Nevada, USA), extracranial and transcranial ultrasound of brain circulation (Phillips HD11XE, Philips Ultrasound, Bothell, WA, USA with linear 3-12 MHz probe), and transthoracic echocardiography (MG) were performed. If the cause of ischaemic stroke had not yet been identified, patients were classified as cryptogenic ischaemic stroke (CIS). Patients selected for the study were enrolled guided by embolic stroke of undetermined source (ESUS) criteria [10]. Only patients with no major-risk cardioembolic source of embolism were included [10]. If no cause of stroke was found related to the event, patients underwent prolonged 14-day ECG-Holter event loop recorder monitoring (MDT Vitaphone Loop 3100 BT, Vitaphone GmbH, Germany) (Fig. 1). Only those patients discharged home or transferred for rehabilitation who understood the Holter monitor operating instructions were included in the study. The 24-hour Holter monitoring was evaluated by a blinded cardiologist (MŠ) via a CardioScan premier 11 (DM software, Nevada, USA). The whole 24-hour recording was reviewed and evaluated. SVE runs were defined as $\geq 3$ consecutive SV beats with an accelerated cycle length lasting < $30 \mathrm{~s}$. The 14-day loop records were evaluated by blinded cardiologists via an external centre (medical data transfer; MDT, Czech Republic).

Statistical analyses were performed using the statistical software SPSS version 25.0 for Windows (SPSS Inc., Chicago, IL, USA). First, a chi-square test was used for a comparison

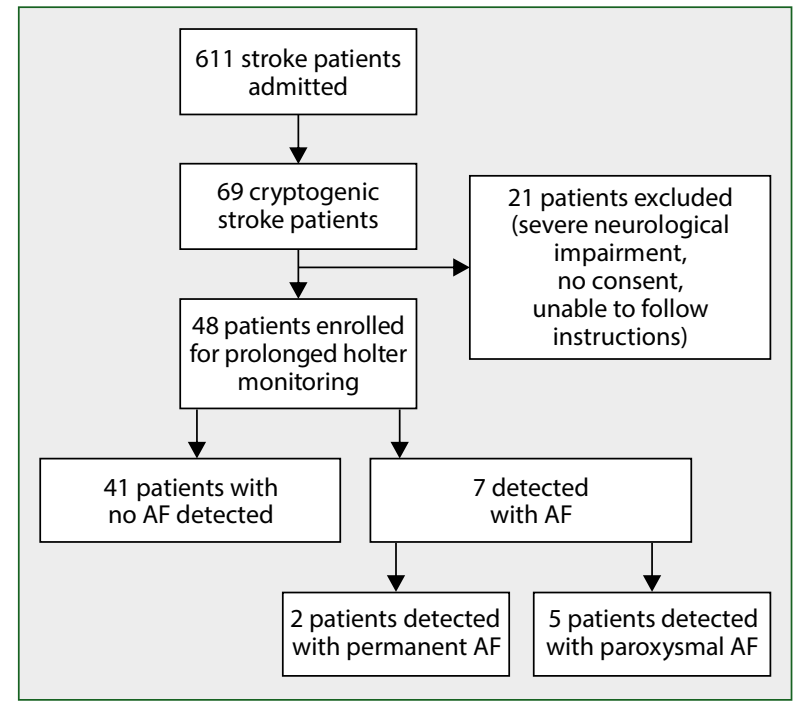

Figure 1. Method flowchart; AF - atrial fibrillation

of categorical variables of the CIS and AF groups. Not normally distributed data were compared using a nonparametric Mann-Whitney U test. Correlation between continuous variables was calculated using Spearman's rank correlation coefficient. Continuous values are reported as mean \pm standard deviation (SD). $\mathrm{P}<0.05$ was considered to indicate a statistically significant difference.

\section{Results}

During the observation period (September 2019-May 2021), we identified 48 patients (mean age $69.9 \pm 8.5$ years, $60.4 \%$ male) diagnosed with CIS who were eligible for prolonged AF screening. The basic characteristics of the patients are set out in Table 1. No statistical differences were observed between the two groups in the baseline variables, except patients with AF had higher admission and discharge NIHSS score $(\mathrm{p}<0.05)$.

AF was detected in seven of the 48 patients (14.6\%) using prolonged 14-day event Holter monitoring initiated a mean of 5.9 days ( \pm 2.3 days) after admission, with no significant interruption during the monitoring. Of these seven patients, five had paroxysmal AF with the mean duration of AF paroxysm of 24.4 hours ( \pm 22.4 hours). The remaining two patients had AF lasting more than seven days, which continued until cessation of ECG monitoring. The mean time from initiation of prolonged monitoring until the first AF detection was 8 days ( \pm 5.1 days, 7-327 hours).

We evaluated the amount of ectopic supraventricular activity based on a 24-hour initial Holter ECG (Tab. 2). Patients with AF were more commonly diagnosed with supraventricular ectopic activity. A significantly higher number of supraventricular extrasystoles (SVES) per hour were found 
Table 1. Demographic data and characteristics of patients with CIS and newly diagnosed AF

\begin{tabular}{llll} 
& \multicolumn{1}{c}{ CIS } & \multicolumn{1}{c}{ AF } & P-value \\
\hline $\mathrm{N}(\mathrm{n})$ & 41 & 7 & \\
Mean age (years \pm SD) & $69.9 \pm 8.8$ & $71 \pm 3.5$ & $\mathrm{~ns}$ \\
Males (\%) & $26(63.4)$ & $3(42.8)$ & $\mathrm{ns}$ \\
Past history of stroke/TIA (\%) & $3(7.3)$ & $1(14.2)$ & $\mathrm{ns}$ \\
Arterial hypertension (\%) & $31(75.6)$ & $5(71.4)$ & $\mathrm{ns}$ \\
Ischaemic heart disease (\%) & $10(24.3)$ & $2(28.5)$ & $\mathrm{ns}$ \\
Myocardial infarction (\%) & $5(12.1)$ & 0 & $\mathrm{~ns}$ \\
Hyperlipidaemia (\%) & $13(31.7)$ & $2(28.5)$ & $\mathrm{ns}$ \\
Diabetes mellitus (\%) & $12(29.2)$ & 0 & $\mathrm{~ns}$ \\
Smoking (\%) & $12(29.2)$ & $1(14.2)$ & $\mathrm{ns}$ \\
Ejection fraction (\% \pm SD) & $54 \pm 6.5$ & $57.5 \pm 4.5$ & $\mathrm{~ns}$ \\
Anterior circulation stroke (\%) & $27(65.8)$ & $4(57.1)$ & $\mathrm{ns}$ \\
Admission NIHSS ( \pm SD) & $4.29 \pm 3.9$ & $5.86 \pm 4.1$ & $<0.05$ \\
Discharge NIHSS ( \pm SD) & $1.38 \pm 1.12$ & $2.71 \pm 3.09$ & $<0.05$ \\
Intravenous thrombolysis (\%) & $8(19.5)$ & $2(28.5)$ & $\mathrm{ns}$ \\
Mechanical thrombectomy (\%) & $5(12.1)$ & 0 & $\mathrm{~ns}$ \\
Cholesterol (mmol/L \pm SD) & $4.8 \pm 1.5$ & $4.1 \pm 0.9$ & $\mathrm{~ns}$ \\
LDL (mmol/L \pm SD) & $3.03 \pm 1.13$ & $2.55 \pm 0.51$ & $\mathrm{~ns}$ \\
HDL (mmol/L \pm SD) & $1.13 \pm 0.44$ & $1.22 \pm 0.33$ & $\mathrm{~ns}$
\end{tabular}

$\mathrm{AF}$ - atrial fibrillation; CIS - cryptogenic ischaemic stroke; HDL — high density lipoproteins; LDL - low density lipoproteins; NIHSS - National Institute of Health Stroke Scale; ns — not significant; $\mathrm{SD}$ - standard deviation; TIA — transitory ischaemic attack

among patients with AF $(150.72 \pm 192.97$ vs. $16.58 \pm 32.3$; $\mathrm{p}<0.022)$. Likewise, a higher number of supraventricular (SV) runs and SV pairs predicted AF in otherwise CIS patients $(\mathrm{p}<0.043 ; \mathrm{p}<0.022$, respectively).

\section{Discussion}

The results of our study confirmed the benefits of early, inpatient prolonged ECG monitoring in patients with cryptogenic stroke. We found that 14-day event Holter monitoring detected a new onset of AF in $14.6 \%$ of patients with cryptogenic stroke. Most of these patients had the paroxysmal type of AF. Our data suggests that excessive supraventricular activity (SV extrasystoles, SV runs and SV pairs) predicts the future occurrence of AF.

Over the past decade, several randomised studies have suggested that prolonged ECG monitoring has led to higher $\mathrm{AF}$ detection, but the results are inconsistent. In the FIND-AF study, AF was found in $12.5 \%$ of patients with cerebral ischaemia using 7-day Holter ECG [11].

In a more recent study with implantable cardiac monitoring (REVEAL-AF), AF detection increased progressively from $6.2 \%$ after 30 days up to $40 \%$ after 30 months, possibly with a higher rate detected if shorter periods ( $<6$ minutes) were included [12]. In contrast, a sub-analysis of the results of the CRYSTAL-AF study implies that only $5.1 \%$ of patients
Table 2. Number of supraventricular ectopic activities during initial 24-hour ECG in CIS and AF patients

\begin{tabular}{llll} 
& \multicolumn{1}{c}{ CIS } & \multicolumn{1}{c}{ AF } & P-value \\
N (n) & 41 & 7 & \\
SVES/hour (mean \pm SD) & $16.58 \pm 32.2$ & $150.72 \pm 192.97$ & $<0.022$ \\
SVES/1,000 beats & $3.93 \pm 7.22$ & $41.59 \pm 54.71$ & $<0.024$ \\
(mean \pm SD) & & & \\
SV run (mean \pm SD) & $3.34 \pm 5.47$ & $83.86 \pm 138.89$ & $<0.043$ \\
SV pair (mean \pm SD) & $6.24 \pm 10.8$ & $259.71 \pm 399.11$ & $<0.022$ \\
IMT ( $\mu$ m \pm SD) & $813 \pm 124$ & $782 \pm 113$ & $\mathrm{~ns}$
\end{tabular}

AF - atrial fibrillation; CIS - cryptogenic ischaemic stroke; ECG - electrocardiogram; IMT intimomedial thickness; ns - not significant; SD — standard deviation; SV — supraventricular; SVES - supraventricular extrasystoles

had a maximum AF in one day of between 2 and 6 minutes, in contrast to $46.2 \%$ with daily AF of between 12 and 24 hours [13]. Also, in our patients the dominant type of AF was paroxysmal (5/7), and none of our cases had an AF paroxysm shorter than one hour. Prolongation of ECG monitoring has also been shown to be important in young ( $<50$ years old) CIS patients, where AF occurred in $7.4 \%$ of CIS patients [14]. One of the reasons for the higher AF detection rate in our study is time to randomisation. Our patients started 14 day event Holter monitoring 5.9 days ( \pm 2.3 days) after their stroke. In the EMBRACE study, prolonged 30 day event triggered recorder increased AF detection rate (16.1\% vs. 3.2\%) compared to conventional 24-hour Holter ECG in patients with CIS. Moreover, half of AF cases were detected during the first week, and three-quarters within two weeks of monitoring. An extension of more than two weeks added an additional $3.2 \%$ of newly detected AF and higher rate of AF detection in patients who started monitoring earlier (18.5\% vs. 9\%) [2].

We found AF in $14.6 \%$ using event triggered recorder during 14 days of monitoring. An explanation for the similar $\mathrm{AF}$ detection rate despite more than doubled monitoring time in the EMBRACE study may lie in the initiation of monitoring. In the EMBRACE study, prolonged (30 days recording) was initiated a mean $75.1 \pm 38.6$ days after the qualifying event, unlike our study where the time from event to the onset of monitoring was 5.9 days ( \pm 2.3 days). This conclusion was also drawn by the study authors as showing relatively long, possibly reducing, sensitivity of AF detection [2]. Likewise, prolonged Holter monitoring was more feasible in young (less than 55 years) CIS patients where 3 -weeks event monitoring revealed AF in seven out of 47 patients. However, time from onset to initiation of monitoring was also rather long (median 42 days) [14].

Early inpatient prolonged and continuous EGC monitoring has also been shown to be feasible in the detection of AF in patients with ESUS. ESUS has been introduced into clinical practice as a subset of patients with cryptogenic stroke for whom a high-risk cardioembolic source has been excluded by the diagnostic process. ESUS-related stroke is a non-lacunar type if no extracranial or intracranial luminal stenosis 
(> 50\%) was present, if no major risk cardioembolic source was found, and if the patient ultimately lacked another specific cause of stroke [10]. Patients with a cryptogenic stroke based on TOAST criteria include patients with incomplete, unknown or more than one possible cause of stroke.

Lumikari et al. [15] detected new onset AF in $12.3 \%$ of ESUS patients during continuous 4 weeks and early (within 8 days) inpatient monitoring. In their study, $85.7 \%$ of AF was revealed within the first week of monitoring. However, they used continous, rather than event triggered, recording, possibly leading to the detection of short bouts of AF.

Regardless of the results of previous studies, we support the use of early inpatient event Holter monitoring in patients with cryptogenic ischaemic stroke, as it leads to higher $\mathrm{AF}$ detection rate and consequently more specific prevention for recurrent stroke. The newly published American Heart Association/American Stroke Association guidelines [16] on stroke prevention in patients with stroke or TIA strongly support early diagnostic evaluation of patients with stroke/ /TIA, including cardiac evaluation, although less evidence is available (IIa, level B-R) regarding the type of monitoring (mobile outpatient, implantable cardiac monitor or other). Currently, screening for AF for at least 72 hours in a patient after stroke/TIA (I, level B) is recommended, and prolonged ECG monitoring should be considered to document AF (IIa, level B) [17]. Some markers may help us select a high-risk patient for AF screening. These include older age with cardiovascular risk factors, cardiac biomarkers and signs of atrial myopathy (supraventricular extrasystoles $>480 / 24$ hours, atrial tachycardia, left atrial diameter $>46 \mathrm{~mm}$ ) and stroke aetiology [18].

There are several lines of evidence to suggest that excessive supraventricular activity determines future AF development. Supraventricular extrasystoles are considered to be a common finding in the general population, but their presence has been found to be associated with an increased risk of stroke, death and cardiovascular disease [19]. Binici et al. [20] reported that ectopic supraventricular activity (SVES and SV runs) in the healthy population was associated with an increased risk of death or stroke and a 2.7-fold increased risk of developing AF. Similarly, Kochhäuser et al. [21] concluded that the number of SVES per hour and of SV runs predict the development of AF. Patients who were later diagnosed with AF had a higher number of SVES compared to patients free of AF (median of 22.8 /hour vs. $0.7 /$ hour respectively). The same authors concluded that an SVES of less than 40 /hour had a $100 \%$ negative predictive value for AF occurrence, unlike an excessive SVES (> 1,660 beats), which predicted AF with $98 \%$ specificity and an $85 \%$ predictive value. This selects the group of patients who need urgent prolongation of ECG monitoring.

In our study, we found that excessive supraventricular activity (SV runs and pairs) is more common in patients diagnosed with AF during prolonged monitoring. Pinho et al. [22] pointed to the number of SVES as a risk factor for the recurrence of TIA and CIS, where frequent SVES
(> 30/hour) were associated with an increased risk of stroke recurrence. However, there is still uncertainty about the optimal threshold for this ectopic activity and its clinical significance. Frequent SVES (> 76/day) have been found to be an independent predictor of mortality and new onset AF (HR 1.38; HR 1.75, respectively) [23]. A similar cut-off value (70 beats/day) as a significant predictor of AF was reported by Wallmann et al. [24]. Patients with frequent FACs (> 70/ /day) had a significantly higher rate of AF detection (26\%vs. 6.5\%; OR 6.6) during subsequent AF screening. Ectopic supraventricular activity ( $>4$ beats/hour $v s .<4$ beats/hour) was associated with a higher prevalence of $\operatorname{AF}(19.6 \%$ vs. $2.8 \%$; p $<0.001)$. Unselected patients with stroke and TIA diagnosed with AF during prolonged Holter monitoring had a higher rate of SVES (median of $29 v s .4$ beats/hours) and the longest SV runs (10 vs. 0 beats; both $\mathrm{p}<0.001)$ [25]. However, the number of SVES per hour differed considerably.

In our study, we have found that in patients without AF, the mean SVES per hour was $16.58 \pm 32.2$, in contrast to patients with AF ( $150.72 \pm 192.97 /$ hour; $\mathrm{p}<0.022)$, which is several times higher than the number per hours in the afore-mentioned studies. In the EMBRACE trial, the number of SVES in patients subsequently diagnosed with AF was 629 beats/24 hours compared to AF-free patients ( 45 beats $/ 24$ hours; $p<0.001$ ). They found that the probability of subsequent detection of AF rose up to 1,500 beats/ 24 hours and reached a higher plateau. The authors also suggested a threshold of $>500$ beats $/ 24$ hours as the definition of frequent atrial premature beats. Suitable candidates for follow-up are patients with even more supraventricular activity ( $>1,000$ beats/ 24 hours) and negative prolonged (4 weeks) Holter monitoring [26].

We have also found that not only isolated SVES or SV runs predict future AF development, but that also the number of SV pairs is significantly $(\mathrm{p}<0.022)$ associated with further AF detection. At present, no exact cut-off value is given for premature/excessive supraventricular beats. However, this should not be a solitary marker for predicting atrial fibrillation, since an echocardiographic marker, such as left atrial size/volume, has also been found to predict AF development [27].

Several limitations to our study need to be addressed. Firstly, a larger sample size would more accurately reflect the association of cryptogenic stroke and excessive supraventricular activity; unfortunately, the COVID-19 pandemic affected our healthcare system and required a rapid reorganisation of inpatient care. Secondly, short or rapid bouts of AF can be missed by external event Holter monitors.

Our study confirmed that early inpatient and prolonged ECG monitoring is feasible in patients with ESUS-related cryptogenic stroke. In addition, in our group of patients, excessive supraventricular activity in patients with cryptogenic ischaemic stroke may predict further AF detection during prolonged monitoring.

Therefore, this otherwise common finding in the general population may be a risk factor in patients with otherwise 
cryptogenic ischaemic stroke, and should be considered in the further diagnostic workup of CIS subjects.

Statement of ethics: This study was performed according to the Declaration of Helsinki of 1975; it was approved by the local ethics committee and all the patients signed written informed consent before enrolling.

Conflict of interest: None.

Funding: Supported by grant VEGA - 1/0380/19.

\section{References}

1. Fonseca AC, Ferro JM, Fonseca AC, et al. Cryptogenic stroke. Eur J Neurol. 2015; 22(4): 618-623, doi: 10.1111/ene.12673, indexed in Pubmed: 25597418.

2. Gladstone DJ, Dorian $P$, Spring $M$, et al. EMBRACE Investigators and Coordinators. Atrial fibrillation in patients with cryptogenic stroke. N Engl J Med. 2014; 370(26): 2467-2477, doi: 10.1056/NEJMoa1311376, indexed in Pubmed: 24963566.

3. Sanna T, Diener HC, Passman RS, et al. CRYSTAL AF Investigators. Cryptogenic stroke and underlying atrial fibrillation. N Engl J Med. 2014; 370(26): 2478-2486, doi: 10.1056/NEJMoa1313600, indexed in Pubmed: 24963567.

4. Marini C, Santis FDe, Sacco S, et al. Contribution of atrial fibrillation to incidence and outcome of ischemic stroke. Stroke. 2005; 36(6): 1115-1119, doi: 10.1161/01.str.0000166053.83476.4a, indexed in Pubmed: 15879330.

5. Engström G, Hedblad B, Juul-Möller S, et al. Cardiac arrhythmias and stroke: increased risk in men with high frequency of atrial ectopic beats. Stroke. 2000; 31(12): 2925-2929, doi: 10.1161/01. str.31.12.2925, indexed in Pubmed: 11108750.

6. Durmaz E, Ikitimur B, Kilickiran Avci B, et al. The clinical significance of premature atrial contractions: how frequent should they become predictive of new-onset atrial fibrillation. Ann Noninvasive Electrocardiol. 2020; 25(3): e12718, doi: 10.1111/anec.12718, indexed in Pubmed: 31603280.

7. Rooney MR, Norby FL, Maheshwari A, et al. Frequent premature atrial contractions are associated with poorer cognitive function in the atherosclerosis risk in communities (ARIC) study. Mayo Clin Proc. 2021; 96(5): 1147-1156, doi: 10.1016/j.mayocp.2021.01.025, indexed in Pubmed: 33840519.

8. Healey JS, Gladstone DJ, Swaminathan B, et al. Recurrent stroke with rivaroxaban compared with aspirin according to predictors of atrial fibrillation: secondary analysis of the NAVIGATE ESUS randomized clinical trial. JAMA Neurol. 2019; 76(7): 764-773, doi: 10.1001/ jamaneurol.2019.0617, indexed in Pubmed: 30958508.

9. Calenda BW, Fuster V, Halperin JL, et al. Stroke risk assessment in atrial fibrillation: risk factors and markers of atrial myopathy. Nat Rev Cardiol. 2016; 13(9): 549-559, doi: 10.1038/nrcardio.2016.106, indexed in Pubmed: 27383079.

10. Hart RG, Diener HC, Coutts SB, et al. Embolic strokes of undetermined source: the case for a new clinical construct. Lancet Neurol. 2014; 13(4): 429-438, doi: 10.1016/s1474-4422(13)70310-7, indexed in Pubmed: 24646875.

11. Stahrenberg R, Weber-Krüger M, Seegers J, et al. Enhanced detection of paroxysmal atrial fibrillation by early and prolonged continuous holter monitoring in patients with cerebral ischemia presenting in sinus rhythm. Stroke. 2010; 41(12): 2884-2888, doi: 10.1161/STROKEAHA.110.591958, indexed in Pubmed: 20966415.

12. Reiffel JA, Verma A, Kowey PR, et al. REVEAL AF Investigators. Incidence of previously undiagnosed atrial fibrillation using insertable cardiac monitors in a high-risk population: the REVEAL AF study. JAMA Cardiol. 2017; 2(10): 1120-1127, doi: 10.1001/jamacardio.2017.3180, indexed in Pubmed: 28842973.

13. Brachmann J, Morillo CA, Sanna T, et al. Uncovering atrial fibrillation beyond short-term monitoring in cryptogenic stroke patients: three-year results from the cryptogenic stroke and underlying atrial fibrillation trial. Circ Arrhythm Electrophysiol. 2016; 9(1): e003333, doi: 10.1161/CIRCEP.115.003333, indexed in Pubmed: 26763225.

14. Sanak D, Hutyra M, Kral M, et al. Paroxysmal atrial fibrillation in young cryptogenic ischemic stroke: a 3-week ECG Holter monitoring study. Biomed Pap Med Fac Univ Palacky Olomouc Czech Repub. 2015; 159(2): 283-287, doi: 10.5507/bp.2015.019, indexed in Pubmed: 25916280.

15. Lumikari TJ, Putaala J, Kerola A, et al. Continuous 4-week ECG monitoring with adhesive electrodes reveals $A F$ in patients with recent embolic stroke of undetermined source. Ann Noninvasive Electrocardiol. 2019; 24(5): e12649, doi: 10.1111/anec.12649, indexed in Pubmed: 31045315.

16. Kleindorfer DO, Towfighi A, Chaturvedi S, et al. 2021 Guideline for the prevention of stroke in patients with stroke and transient ischemic attack: a guideline from the American Heart Association/American Stroke Association. Stroke. 2021; 52(7): e364-e467, doi: 10.1161/ STR.0000000000000375, indexed in Pubmed: 34024117.

17. Hindricks G, Potpara T, Dagres N, et al. ESC Scientific Document Group. 2020 ESC Guidelines for the diagnosis and management of atrial fibrillation developed in collaboration with the European Association for Cardio-Thoracic Surgery (EACTS): the Task Force for the diagnosis and management of atrial fibrillation of the European Society of Cardiology (ESC) developed with the special contribution of the European Heart Rhythm Association (EHRA) of the ESC. Eur Heart J. 2021; 42(5): 373-498, doi: 10.1093/eurheartj/ehaa612, indexed in Pubmed: 32860505.

18. Schnabel RB, Haeusler KG, Healey JS, et al. Searching for atrial fibrillation poststroke: a white paper of the AF-SCREEN International Collaboration. Circulation. 2019; 140(22): 1834-1850, doi: 10.1161/ CIRCULATIONAHA.119.040267, indexed in Pubmed: 31765261.

19. Huang BT, Huang FY, Peng Y, et al. Relation of premature atrial complexes with stroke and death: systematic review and meta-analysis. Clin Cardiol. 2017; 40(11): 962-969, doi: 10.1002/clc.22780, indexed in Pubmed: 28846809.

20. Binici Z, Intzilakis T, Nielsen OW, et al. Excessive supraventricular ectopic activity and increased risk of atrial fibrillation and stroke. Circulation. 2010; 121(17): 1904-1911, doi: 10.1161/CIRCULATIONAHA.109.874982, indexed in Pubmed: 20404258.

21. Kochhäuser S, Dechering DG, Dittrich R, et al. Supraventricular premature beats and short atrial runs predict atrial fibrillation in continuously monitored patients with cryptogenic stroke. Stroke. 2014; 45(3): 884-886, doi: 10.1161/STROKEAHA.113.003788, indexed in Pubmed: 24425117.

22. Pinho J, Braga CG, Rocha S, et al. Atrial ectopic activity in cryptogenic ischemic stroke and TIA: a risk factor for recurrence. J Stroke Cerebrovasc Dis. 2015; 24(2): 507-510, doi: 10.1016/j.jstrokecerebrovasdis.2014.09.029, indexed in Pubmed: 25533759. 
23. Lin CY, Lin YJ, Chen YY, et al. Prognostic significance of premature atrial complexes burden in prediction of long-term outcome. J Am Heart Assoc. 2015; 4(9): e002192, doi: 10.1161/JAHA.115.002192, indexed in Pubmed: 26316525.

24. Wallmann D, Tüller D, Wustmann $\mathrm{K}$, et al. Frequent atrial premature beats predict paroxysmal atrial fibrillation in stroke patients: an opportunity for a new diagnostic strategy. Stroke. 2007; 38(8): 2292 2294, doi: 10.1161/STROKEAHA.107.485110, indexed in Pubmed: 17585079 .

25. Weber-Krüger $M$, Gröschel $K$, Mende $M$, et al. Excessive supraventricular ectopic activity is indicative of paroxysmal atrial fibril- lation in patients with cerebral ischemia. PLoS One. 2013; 8(6): e67602, doi: 10.1371/journal.pone.0067602, indexed in Pubmed: 23840747.

26. Gladstone DJ, Dorian P, Spring M, et al. EMBRACE Steering Committee and Investigators. Atrial premature beats predict atrial fibrillation in cryptogenic stroke: results from the EMBRACE trial. Stroke. 2015; 46(4): 936-941, doi: 10.1161/STROKEAHA.115.008714, indexed in Pubmed: 25700289.

27. Kamel H, Okin PM, Elkind MSV, et al. Atrial fibrillation and mechanisms of stroke: time for a new model. Stroke. 2016; 47(3): 895-900, doi: 10.1161/STROKEAHA.115.012004, indexed in Pubmed: 26786114. 